\title{
UNIDIRECTIONALIZATION OF HAMILTONIAN WAVES
}

\author{
Peter J. OLVER ${ }^{1}$ \\ School of Mathematics, University of Minnesota, Minneapolis, MN 55455, USA
}

Received 28 September 1987; accepted for publication 24 November 1987

Communicated by D.D. Holm

\begin{abstract}
We show how the restriction of certain bidirectional hamiltonian systems modelling nonlinear, one-dimensional wave propagation to waves moving in a single direction preserves the hamiltonian structure, even though the perturbation expansion of the bidirectional hamiltonian is not correct. A combination of the two approaches of direct hamiltonian perturbation theory and the method of multiple scales helps explain the apperance of integrable bihamiltonian wave models.
\end{abstract}

The most famous example of "unidirectionalization" is the derivation of the Korteweg-de Vries equation as a model equation for the unidirectional propagation of long waves in shallow water. There are two basic perturbation methods which lead to this model. The classical Boussinesq expansion (cf. ref. [1], § 13.11) rests on the direct introduction of a small parameter $\epsilon$ into the problem, and then truncating the resulting perturbation expansion at order $\epsilon$. An alternative method, based on multiple time scales and the suppression of secular terms has been used by Ablowitz and Segur (see ref. [2], § 4.1a). The multiple scales method has the advantage that Zakharov's hamiltonian structure of the full water wave system [3] immediately restricts to the first (Gardner) hamiltonian structure of the Korteweg-de Vries model. On the other hand, Olver $[4,5]$ noticed that since the Boussinesq expansion is not canonical, the direct perturbation expansion does not preserve the water wave hamiltonian structure, and instead leads to a lincar combination of the two hamiltonian structures of the Korteweg-de Vries equation itself. In this paper, we reconcile the two perturbation theories by showing how general unidirectional models inherit their first hamiltonian structure from the hamiltonian structure of the corresponding bidirectional (Boussinesq) model via the

1 Research supported in part by NSF Grant DMS 86-02004 and NATO Collaborative Research Grant RG 86/0055. multiple scales method, and, moreover, in many cases acquire an additional hamiltonian structure from the direct hamiltonian perturbation method. Thus, as argued earlier in ref. [5], a wide variety of unidirectional wave models arising from noncanonical perturbation expansions of physical systems are automatically bihamiltonian systems, and thereby can be viewed as "completely integrable" according to Magri's theorem (see ref. [6] and ref. [7], theorem 7.24). This helps further explain the remarkable preponderance of soliton equations as the model equations for such a wide variety of physical systems. In either perturbation method, the key phase in understanding the loss or the retention of hamiltonian structure is the specialization from a bidirectional Boussinesq model to the unidirectional Korteweg-de Vries model. (The multiple scales method usually manages to bypass the Boussinesq models, but the key issue is most easily seen without reverting back to the full free boundary problem for water wavcs.) Therefore, we will concentrate on the unidirectionalization of bidirectional wave models like the Boussinesq system.

Consider a general evolutionary system in hamiltonian form,

$\boldsymbol{u}_{t}=\mathscr{D} \cdot E_{\boldsymbol{u}}(H)$,

where $\mathscr{D}$ is the hamiltonian operator, $\mathscr{H}[\boldsymbol{u}]=\int H[\boldsymbol{u}] \mathrm{d} x$ the hamiltonian functional, and $E_{u}$ the Euler operator or variational derivative (cf. 
ref. [7], ch. 7). In the cases of interest here, $x \in \mathbb{R}$,

$\boldsymbol{u}(x, t)=\left(\begin{array}{l}u(x, t) \\ v(x, t)\end{array}\right) \in \mathbb{R}^{2}$,

the hamiltonian operator is the matrix differential operator

$\left(\begin{array}{cc}0 & D_{x} \\ D_{x} & 0\end{array}\right)$

and the hamiltonian density $H[u]$ is a smooth function of $u$ and $v$ and their derivatives with respect to $x$, which we denote by $u_{i}=\partial^{i} u / \partial x^{i}$. The system constitutes a two-dimensional hyperbolic system of conservation laws,

$u_{t}=D_{x} E_{v}(H), \quad v_{t}=D_{x} E_{u}(H)$.

Such models arise in gas dynamics [8] and viscoelasticity [9], but we will concentrate on those which model bidirectional wave propagation. Usually these come themselves from a perturbation expansion of the full physical problem, e.g. the Boussinesq equations for the problem of surface waves. Let $\epsilon$ denote the small parameter in the problem. (In the Korteweg-de Vries equation, $\epsilon$ is proportional to the ratios of wave amplitude to depth and of depth squared to wave length squared.) Normalizing the linearized wave speed to 1 , we postulate the system be in the form

$u_{t}+v_{x}+\epsilon D_{x} E_{v}(H)=0$,

$v_{t}+u_{x}+\epsilon D_{x} E_{u}(H)=0$,

where the hamiltonian density is now

$\mathscr{H}[\boldsymbol{u}]=-\int\left(\frac{1}{2} u^{2}+\frac{1}{2} v^{2}+\epsilon H[u, v]\right) \mathrm{d} x$.

Some, but not all, of the variants of the Boussinesq equations $[4,10]$ are of this type. A particularly interesting example is the canonical Boussinesq equation

$u_{t}+v_{x}+\epsilon \mathfrak{u} u_{x}=0$,

$v_{t}+u_{x}+\epsilon(u v)_{x}+\epsilon u_{x x x}=0$,

in which the hamiltonian takes the form (4) with $H[u, v]=\frac{1}{2} u^{2} v-\frac{1}{2} u_{x}^{2}$. (Kupershmidt [11] showed that (4) is a trihamiltonian system, and Kaup [12] showed how it could be integrated by inverse scattering techniques.)
To leading order in $\epsilon,(3)$ is just the linear wave equation, with solution

$u=\varphi(\xi)+\psi(\eta), \quad v=\varphi(\xi)-\psi(\eta)$,

where $\xi=x-t, \eta=x+t$ are the characteristic variables, representing waves moving in both directions. We can extract the waves moving to the right by setting $v=u$ in the linear system. We therefore specialize to a nonlinear submanifold of unidrectional solutions by postulating a perturbation expansion of the form

$v=u+\epsilon P[u]+\ldots$.

Plugging (6) into (3), and expanding, we find the two equations reduce to

$u_{t}+u_{x}+\epsilon\left[D_{x} P+\left.D_{x} E_{v}(H)\right|_{u=v}\right]+\mathrm{O}\left(\epsilon^{2}\right)=0$,
$u_{t}+u_{x}+\epsilon\left[D_{t} P+\left.D_{x} E_{u}(H)\right|_{u=\imath}\right]+\mathrm{O}\left(\epsilon^{2}\right)=0$.

The goal is to choose the nonlinear term $P[u]$ in (6) so as to make both equations in (7) agree up to order $\epsilon$. Now

$$
\begin{aligned}
D_{t} P & =\sum_{i} \frac{\partial P}{\partial u_{i}} u_{i, t}=-\sum_{i} \frac{\partial P}{\partial u_{i}} u_{i+1}+O(\epsilon) \\
& =-D_{x} P+O(\epsilon),
\end{aligned}
$$

so the two equations in (7) will agree up to order $\epsilon$ provided

$D_{x}\left[P+\left.E_{v}(H)\right|_{u=v}\right]=D_{x}\left[-P+\left.D_{x} E_{u}(H)\right|_{u=v}\right]$.

Consequently, we are led to the choice

$P[u]=\left.\frac{1}{2}\left[E_{u}(H)-E_{v}(H)\right]\right|_{u=v}$

as the nonlinear correction. Then (7) reduces to the common unidirectional model

$u_{t}+u_{x}+\left.\frac{1}{2} \epsilon D_{x}\left[E_{u}(H)+E_{v}(H)\right]\right|_{u=l}=0$.

Note that (9) is automatically in hamiltonian form (1), where

$\mathscr{D}=\mathscr{D}_{0}=D_{x}$

is the Gardner hamiltonian operator, and the hamiltonian functional is

$\tilde{\mathscr{H}}[u]=\int\left(\frac{1}{2} u^{2}+\left.\frac{1}{2} \epsilon H\right|_{u=v}\right) \mathrm{d} x$.

Indeed, we find 


$$
\begin{aligned}
& {\left.\left[E_{u}(H)+E_{v}(H)\right]\right|_{u=v}} \\
& \quad=\left.\sum\left(-D_{x}\right)^{k}\left(\frac{\partial H}{\partial u_{k}}+\frac{\partial H}{\partial v_{k}}\right)\right|_{u=v} \\
& \quad=\sum\left(-D_{x}\right)^{k} \frac{\partial}{\partial u_{k}}\left(\left.H\right|_{u=v}\right)=E_{u}\left(\left.H\right|_{u=v}\right) .
\end{aligned}
$$

For example, consider the canonical Boussinesq equations (5). Here $H[u, v]=\frac{1}{2} u^{2} v-\frac{1}{2} u_{x}^{2}$, so $P[u]=$ $\frac{1}{4} u^{2}+\frac{1}{2} u_{x x}$, and (9) becomes the Korteweg-de Vries equation

$u_{\tau}+u_{x}+\frac{3}{2} \epsilon u u_{x}-\frac{1}{2} \epsilon u_{x x x}=0$,

written in the original Gardner hamiltonian form with the hamiltonian $\tilde{H}[u]=\frac{1}{2} u^{2}+\frac{1}{4} \epsilon u^{3}-\frac{1}{4} \epsilon u_{x}^{2}$ a linear combination of two of the classical conservation laws.

The direct approach always leads to the same model as the multiple scales method used by Ablowitz and Segur [2]. In this method, starting with the bidirectional system (3), we introduce a "slow time" $\tau=\epsilon t$, and writc $u=u(x, t, \tau), v=v(x, t, \tau)$, so $u_{t}$ and $v_{1}$ get replaced by $u_{t}+\epsilon u_{\tau}$ and $v_{t}+\epsilon v_{\tau}$. Thus (3) becomes

$u_{i}+v_{x}+\epsilon\left[u_{\tau}+D_{x} E_{\nu}(H)\right]=0$,

$v_{t}+u_{x}+\epsilon\left[v_{\tau}+D_{x} E_{u}(H)\right]=0$.

If we now expand

$u=u^{0}+\epsilon u^{1}+\ldots, \quad v=v^{0}+\epsilon v^{1}+\ldots$,

then the leading order terms are

$u_{i}^{0}+v_{x}^{0}=0, v_{t}^{0}+u_{x}^{0}=0$,

with solution

$u^{0}=\varphi(\xi, \tau)+\psi(\eta, \tau), \quad v^{0}=\varphi(\xi, \tau)-\psi(\eta, \tau)$,

as before. Substituting into (11), we find that at order $\epsilon$, the secular term in $\xi$ is

$$
\begin{aligned}
\varphi_{\tau} & +\left.\frac{1}{2} \epsilon D_{\xi}\left[E_{u}(H)+E_{\nu}(H)\right]\right|_{u=v=\varphi} \\
& =\varphi_{\tau}+\frac{1}{2} \epsilon D_{\xi} E_{\varphi}\left(\left.H\right|_{u=v=\varphi}\right) .
\end{aligned}
$$

(There is a similar secular term in $\eta$, which will lead to an identical model for the waves moving to the left.) To eliminate the secular term, we require that it vanish, so we deduce that $\varphi$ must be a solution to the equation $\varphi_{\tau}+\frac{1}{2} \epsilon D_{\xi} E_{\varphi}\left(\left.H\right|_{u=v=\varphi}\right)=0$,

which is clearly hamiltonian with respect to the hamiltonian operator $D_{\xi}$. However, (12) agrees with the direct perturbation model (9) if we set

$x=\xi+\tau / \epsilon, \quad t=\tau / \epsilon, \quad \varphi=u$.

Thus we deduce that the reason the direct perturbation model is always hamiltonian is the same reason why the identical multiple scales model is also hamiltonian.

However, we should remark that all of the above remarks depend crucially on the form of the initial hamiltonian operator; if the bidirectional model (3) has a different hamiltonian structure, then we have no reason to expect the first order model (either direct or multiple scales expansion) to be hamiltonian. The simplest example I could think of where this occurs is to take

$\mathscr{D}=\left(\begin{array}{cc}2 v D_{x}+v_{x} & 2(u+1) D_{x}+u_{x} \\ 2(u+1) D_{x}+u_{x} & 2 v D_{x}+v_{x}\end{array}\right)$,

which is (if we replace $u+1$ by $u$ ) the second hamiltonian operator for the shallow water equations, a special case of polytropic gas dynamics when the exponent $\gamma=3[8]$ and

$\mathscr{H}[u, v]=-\int\left[u-\epsilon\left(\frac{1}{2} v_{x}^{2}+\frac{1}{6} u_{x}^{3}\right)\right] \mathrm{d} x$.

The bidirectional system is

$$
\begin{aligned}
& u_{t}+v_{x}+\epsilon\left(2 v_{x x x}+2 u v_{x x x}+u_{x} v_{x x}+2 u_{x} u_{x x x} v\right. \\
& \left.\quad+2 u_{x x}^{2} v+u_{x} u_{x x} v_{x}\right)=0, \\
& v_{t}+u_{x}+\epsilon\left(2 u_{x} u_{x x x}+2 u_{x x}^{2}+2 u u_{x} u_{x x x}+2 u u_{x x}^{2}\right. \\
& \left.\quad+u_{x}^{2} u_{x x}+2 v v_{x x x}+v_{x} v_{x x}\right)=0 .
\end{aligned}
$$

In this case $P[u]=-u_{x x}+u_{x} u_{x x}$ in (6), and the common unidirectional model is

$$
\begin{aligned}
& u_{t}+u_{x}+\epsilon\left(u_{x x x}+2 u u_{x x x}+u_{x} u_{x x}+2 u u_{x} u_{x x x}\right. \\
& \left.\quad+2 u u_{x x}^{2}+u_{x}^{2} u_{x x}+u_{x} u_{x x x}+u_{x x}^{2}\right)=0
\end{aligned}
$$

which has no obvious hamiltonian structure or even conserved density to serve as the hamiltonian. (The multiple scales method will of course lead to the same unidirectional equation.) Many other examples of this type can be readily constructed.

We now compare with the hamiltonian perturba- 
tion theory of refs. [4,5]. In this approach, we expand both the hamiltonian operator

$\mathscr{D} \rightarrow \mathscr{D}_{0}+\epsilon \mathscr{D}_{1}+\ldots$,

and the hamiltonian functional

$\mathscr{H}=\mathscr{H}_{0}+\epsilon \mathscr{H}_{1}+\ldots$.

and truncate at order $\epsilon$. (One difficulty is that since the Jacobi conditions on $\mathscr{D}$ are nonlinear, the truncated operator $\hat{\mathscr{D}}=\mathscr{D}_{0}+\epsilon \mathscr{D}_{1}$ may not be hamiltonian.) The resulting (pseudo-)hamiltonian model

$$
\begin{aligned}
u_{t} & =\hat{\mathscr{D}} \cdot E_{u}(\hat{H})=\left(\mathscr{D}_{0}+\epsilon \mathscr{D}_{1}\right) \cdot E_{u}\left(H_{0}+\epsilon H_{1}\right) \\
& =\mathscr{D}_{0} \cdot E_{u}\left(H_{0}\right)+\epsilon\left[\mathscr{D}_{0} \cdot E_{u}\left(H_{1}\right)+\mathscr{D}_{1} \cdot E_{u}\left(H_{0}\right)\right] \\
& +\epsilon^{2} \mathscr{D}_{1} \cdot E_{u}\left(H_{1}\right)
\end{aligned}
$$

retains some, but not all terms of order $\epsilon^{2}$. In our particular case, on the unidirectional submanifold (6), the hamiltonian itself has expansion

$$
\mathscr{H}[u]=\frac{1}{2} \int\left(u^{2}+\left.\epsilon H\right|_{u=1}+\epsilon u \cdot P[u]\right) \mathrm{d} x+O\left(\epsilon^{2}\right),
$$

the factor of $\frac{1}{2}$ coming from the fact that we are restricting to a submanifold (cf. ref. [5]). We then truncate to first order; the perturbed hamiltonian is $\hat{H}[u]=H_{0}[u]+\epsilon \hat{H}_{1}[u]$, where

$H_{0}[u]=\frac{1}{2} u^{2}$,

$\hat{H}_{1}[u]=\left.\frac{1}{2} H[u, v]\right|_{u=u^{\prime}}+\frac{1}{2} u \cdot P[u]$.

Note that $\hat{H}[u]$ does not agree with the hamiltonian $\tilde{H}[u]$ coming from the direct expansion, cf. (10), unless $P[u]$, as given by (8), vanishes! In the Korteweg-de Vries example, as noted in refs. [4,5], the correct expansion of the water wave energy is

$\hat{H}[u]=\frac{1}{2} u^{2}+\frac{3}{8} \epsilon u^{3}-\frac{1}{4} \epsilon u_{x}^{2}-\frac{1}{4} \epsilon u u_{x x} \approx \frac{1}{2} u^{2}+\frac{3}{8} \epsilon u^{3}$

(the omitted terms form a divergence), which does not agree with the Gardner hamiltonian, or, indeed, with any of the conservation laws of the Korteweg-de Vries equation.

To obtain the corresponding perturbed hamiltonian operator, we expand the associated Poisson bivector (cf. ref. [7], ch. 7)

$\Theta=\frac{1}{2} \int \xi \wedge D_{.} \eta \mathrm{d} x$,

where $\xi$ and $\eta$ are the "uni-vectors" dual to $\mathrm{d} u$ and $\mathrm{d} v$. Using the rule (3.2) in ref. [5], if $v$ is given by (6), then

$\xi=\eta+\epsilon D_{P}^{*} \eta+\mathrm{O}\left(\epsilon^{2}\right)$,

where $D_{P}^{*}$ denotes the adjoint of the Fréchet derivative of $P$ (see ref. [7], p. 323) hence

$\eta=\xi-\epsilon D_{P}^{*} \xi+\mathrm{O}\left(\epsilon^{2}\right)$.

Substituting, we find that

$\Theta=\frac{1}{2} \int \xi \wedge D_{x}\left[\xi-\epsilon D_{P}^{*} \xi+\mathrm{O}\left(\epsilon^{2}\right)\right] \mathrm{d} x$.

Skew-symmetrizing, we find the perturbed hamiltonian operator, is, to first order,

$\hat{\mathscr{D}}=D_{x}-\frac{1}{2} \epsilon\left(D_{P} \cdot D_{x}+D_{x} \cdot D_{P}^{*}\right) \equiv \mathscr{D}_{0}-\frac{1}{2} \epsilon \mathscr{D}_{1}$.

Thus we see the occurrence both of the Gardner hamiltonian operator $\mathscr{Z}_{0}=D_{x}$, as well as another skew-adjoint operator $\mathscr{D}_{1}=D_{P} \cdot D_{x}+D_{x} \cdot D_{P}^{*}$. In many cases, $\mathscr{D}_{1}$ is actually a hamiltonian operator; for instance, in the Korteweg-de Vries example, $\mathscr{D}_{1}=-D_{x}^{3}+u D_{x}+\frac{1}{2} u_{x}$ is the second hamiltonian structure. The hamiltonian unidirectional model (13) is

$$
\begin{aligned}
u_{t} & =\mathscr{D}_{0} E_{u}\left(I_{0}\right)+\epsilon\left[\mathscr{D}_{0} E_{u}\left(I_{1}\right)-\frac{1}{2} \mathscr{D}_{1} E_{u}\left(I_{0}\right)\right] \\
& -\frac{1}{2} \epsilon^{2} \mathscr{D}_{1} E_{u}\left(H_{1}\right) .
\end{aligned}
$$

It should be emphasized that the model equations (9) and (15) are exactly the same up to order $\epsilon$ (as they have to be), so

$\mathscr{D}_{0} E_{u}\left(H_{0}\right)=D_{x}(u)=u_{x}$,

while the quantity

$$
\begin{aligned}
& \mathscr{P}_{0} E_{u}\left(H_{1}\right)-\frac{1}{2} \mathscr{Q}_{1} E_{u}\left(H_{0}\right) \\
& \quad=D_{x}\left\{\frac{1}{2} E_{u}\left(\left.H\right|_{u=v}+u \cdot P[u]\right)\right\} \\
& -\frac{1}{2}\left(D_{P} \cdot D_{x}+D_{x} \cdot D_{P}^{*}\right) u
\end{aligned}
$$

must agree with the order $\epsilon$ terms in (9), which are $\frac{1}{2} D_{x} E_{u}\left(\left.H\right|_{u=v}\right)$.

Thus we conclude that

$D_{x}(u \cdot P[u])=\left(D_{P} \cdot D_{x}+D_{x} \cdot D_{P}^{*}\right) u$,

an identity which can, in fact, be verified by direct computation.

Summarizing, we have shown that the order $\epsilon$ unidirectional wave model for the bidirectional system 
can be written in two different forms. The multiple scales approach shows that it is automatically in hamiltonian form,

$u_{t}=\mathscr{D}_{0} \cdot E_{u}\left(H_{0}\right)+\epsilon \mathscr{D}_{0} \cdot E_{u}\left(\tilde{H}_{1}\right)$,

$\tilde{H}_{1}=\left.\frac{1}{2} \epsilon H\right|_{u=\nu}$.

On the other hand, the hamiltonian perturbation method shows that it is in the form

$u_{t}=\mathscr{D}_{0} \cdot E_{u}\left(H_{0}\right)+\epsilon\left[\mathscr{D}_{0} \cdot E_{u}\left(\hat{H}_{1}\right)-\frac{1}{2} \mathscr{D}_{1} \cdot E_{u}\left(H_{0}\right)\right]$,

$\hat{H}_{1}=\tilde{H}_{1}+\frac{1}{2} u \cdot P[u]$.

These are the same equation, so the order $\epsilon$ terms must agree; therefore

$\mathscr{D}_{0} \cdot E_{u}\left(H_{1}^{*}\right)=-\frac{1}{2} \mathscr{D}_{1} \cdot E_{u}\left(H_{0}\right)$,

$H_{1}^{*}=\frac{1}{2} u \cdot P[u]$,

an equation that is reminiscent of the bihamiltonian condition of Magri. We do not have any guarantee that the skew-adjoint operator $\mathscr{D}_{1}$ is actually hamiltonian; however, in many cases, we can assert that the unidirectional model (9) is actually a bihamiltonian system, and hence "completely integrable" by Magri's theorem.

Proposition. If the skew-adjoint operator

$\mathscr{D}_{1}=D_{P} \cdot D_{x}+D_{x} \cdot D_{P}^{*}$

is hamiltonian, then $\mathscr{D}_{0}$ and $\mathscr{D}_{1}$ form a hamiltonian pair (see ref. [7], definition 7.19).

Proof. According to ref. [7], corollary 7.21, we must check a compatibility condition between the bivectors

$\boldsymbol{\theta}_{0}=\frac{1}{2} \int \xi \wedge D_{x} \xi \mathrm{d} x$

and

$$
\begin{aligned}
\Theta_{1} & =\frac{1}{2} \int \xi \wedge\left(D_{P} \cdot D_{x}+D_{x} \cdot D_{P}^{*}\right) \xi \mathrm{d} x \\
& =\int \xi \wedge D_{P} \cdot \xi_{x} \mathrm{~d} x
\end{aligned}
$$

Now $\theta_{0}$ is independent of $u$, so the compatibility condition reduces to

$$
\begin{gathered}
\left.\operatorname{pr} v_{v_{0} \xi}\left(\Theta_{1}\right)=-\int \xi \wedge \operatorname{pr} v_{\xi_{x}}\left(D_{P}\right) \wedge \xi_{x}\right) \mathrm{d} x \\
\quad=-\int \xi \wedge D_{P}^{2}\left(\xi_{x} \wedge \xi_{x}\right) \mathrm{d} x=0 .
\end{gathered}
$$

But this is trivial, since the second Fréchet derivative $D_{P}^{2}$ is a symmetric operator.

It is thus of great interest to determine when a differential operator of the form (19) is indeed hamiltonian, i.e. when does it satisfy the Jacobi identity. The computational techniques introduced in refs. $[7,13,14]$ should aid in answering this question.

In the case when (19) is a hamiltonian operator, then (subject to the usual technical hypotheses in Magri's theorem), the hamiltonians $H_{0}$ and $H_{1}^{*}$ form the first two terms in an infinite hierarchy of commuting hamiltonians and hamiltonian flows,

$u_{t}=\mathscr{D}_{0} \cdot E_{u}\left(H_{n}^{*}\right)=\mathscr{D}_{1} \cdot E_{u}\left(H_{n-1}^{*}\right)$.

However, since $\tilde{H}_{1}$ is not quite the same as $H_{1}^{*}$, the unidirectional model (16) does not quite fit into this hierarchy. In many special cases, the original hamiltonian $H[u, v]$ is a homogeneous polynomial in $u$ and $v$, i.e. $H[\lambda u, \mu v]=\lambda^{m} \mu^{n} \cdot H[u, v]$, and, consequently, $\tilde{H}_{1}$ equals a multiple of $H_{1}^{*}$, so (16) itself is a bihamiltonian system.

Theorem. Suppose the skew-adjoint operator $\mathscr{D}_{1}$ is hamiltonian and the hamiltonian $H[u, v]$ is homogeneous. Then the unidirectional model equation (16) lies in an integrable bihamiltonian hierachy.

Proof. We first note the simple integration by parts result that for any hamiltonian $H[u]$, the expression $u \cdot E_{u}(H)$ differs from

$N_{u}(H)=\sum_{i} u_{i} \frac{\partial H}{\partial u_{i}}$

by a divergence. But if $H[u]$ is a homogeneous function of $u$ and its derivatives, then Euler's theorem implies that the operator $N_{u}$ just multiplies $H$ by its degree of homogeneity. Therefore, if $H[u, v]$ is homogeneous in $u$ and $v$,

$2 H_{1}^{*}[u]=u \cdot P[u]=\left.u\left[E_{u}(H)-E_{v}(H)\right]\right|_{u=v}$

differs from a multiple of $\tilde{H}_{1}=\left.\frac{1}{2} \epsilon H\right|_{u=v}$ by a divergence, and hence serves as an equivalent hamiltonian density for the system. Thus the bihamiltonian condition (18) implies that (16) is just a linear combination of the first two flows in the bihamiltonian hierarchy (20).

I would like to thank Harvey Segur, who originally raised the questions concerning the earlier results on 
hamiltonian perturbation theory that led to this paper.

\section{References}

[1] G,B. Whitham, Linear and nonlinear waves (Wiley, New York, 1974).

[2] M.J. Ablowitz and H. Segur, Solitons and the inverse scattering transform (SIAM, Philadelphia, 1981).

[3] V.E. Zakharov, J. Appl. Math. Tech. Phys. 2 (1968) 190.

[4] P.J. Olver, Hamiltonian and non-hamiltonian models for water waves, in: Springer lecture notes in physics, Vol. 195. Trends and applications of pure mathematics to mechanics, eds. P.G. Ciarlet and M. Roseau (Springer, Berlin, 1984) pp. 273-290.
[5] P.J. Olver, Contemp. Math. 28 (1984) 231.

[6] F. Magri, J. Math. Phys. 19 (1978) 1156.

[7] P.J. Olver, Graduate texts in mathematics, Vol. 107. Applications of Lie groups to differential equations (Springer, Berlin, 1986).

[8] Y. Nutku, On a new class of completely integrable systems. II. Multi-hamiltonian structure, preprint.

[9] C.M. Dafermos, SIAM J. Math. Anal. 18 (1987) 409.

[10] J.L. Bona and R. Smith, Math. Proc. Cambridge Philos. Soc. 79 (1976) 167.

[11] BA. Kupershmidt, Commun. Math. Phys. 99 (1985) 51.

[12] D.J. Kaup, Prog. Theor. Phys. 54 (1975) 396.

[13] A.M. Astashov and A.M. Vinogradov, J. Geom. Phys. 3 (1986) 263.

[14] P.J. Olver, Darboux' theorem for hamiltonian differential operators, J. Diff. Eq., to be published. 